\title{
Dynamic stress and displacement in an elastic half-space with a cylindrical cavity
}

\author{
İ. Coşkun ${ }^{\mathrm{a}, *}, \mathrm{H}$. Engin ${ }^{\mathrm{b}}$ and A. Özmutlu ${ }^{\mathrm{c}}$ \\ ${ }^{a}$ Faculty of Civil Engineering, Ylldız Technical University, Davutpaşa Campus, 34210 Esenler, Istanbul, Turkey \\ ${ }^{\mathrm{b}}$ Faculty of Civil Engineering, Istanbul Technical University, 34469 Maslak, Istanbul, Turkey \\ ${ }^{\mathrm{c}}$ Department of Civil Engineering, Trakya University, 2250 Karaağaç, Edirne, Turkey
}

Received 8 February 2010

Revised 2 June 2010

\begin{abstract}
The dynamic response of an elastic half-space with a cylindrical cavity in a circular cross-section is analyzed. The cavity is assumed to be infinitely long, lying parallel to the plane-free surface of the medium at a finite depth and subjected to a uniformly distributed harmonic pressure at the inner surface. The problem considered is one of plain strain, in which it is assumed that the geometry and material properties of the medium and the forcing function are constant along the axis of the cavity. The equations of motion are reduced to two wave equations in polar coordinates with the use of Helmholtz potentials. The method of wave function expansion is used to construct the displacement fields in terms of the potentials. The boundary conditions at the surface of the cavity are satisfied exactly, and they are satisfied approximately at the free surface of the half-space. Thus, the unknown coefficients in the expansions are obtained from the treatment of boundary conditions using a collocation least-square scheme. Numerical results, which are presented in the figures, show that the wave number (i.e., the frequency) and depth of the cavity significantly affect the displacement and stress.
\end{abstract}

Keywords: Dynamic response, half-space, cavity, wave function, least-squares technique

\section{Introduction}

The problem of the dynamic response of an elastic medium that contains a cavity or shell-like structure has been the subject of numerous studies. Underground pipelines, tunnels and underwater pipelines have been the major subjects of this problem. Because of the mathematical simplicity of modeling, the dynamic response of an infinite medium involving a cavity has widely been studied in the literature, including textbooks by Achenbach [1], Eringen and Şuhubi [2], Graff [3] and Pao and Mow [4]. Compared with the large volume of literature on the dynamic response of structures in an infinite medium, the corresponding problem in a half-space has not received much attention, although it is more suitable for application. The authors presume that this limitation is mainly due to difficulties in satisfying the boundary conditions at the free surface of the half-space. Thiruvenkatachar and Viswanathan [5] investigated the dynamic response of an elastic half-space with a cylindrical cavity at a finite depth subjected to time-dependent surface tractions on the boundary of the cavity. The solution was obtained by expanding the field in a series of wave functions and applying the method of successive approximations. El-Akily and Datta [6,7] studied the response of a circular cylindrical shell to disturbances in an elastic half-space. The external field represented by a series expansion in cylindrical wave functions, whereas the shell was modeled using Flügge's bending theory. Matched asymptotic expansions and a successive reflection technique were used to determine the coefficients in the expansion. Datta et al. [8] studied the dynamic response of a cylindrical pipe of circular cross-section lying in a

${ }^{*}$ Corresponding author. E-mail: coskun @yildiz.edu.tr. 
concentric cylindrical region buried in an elastic half-space. The fields within each of the regions were expanded in a series of wave functions. The unknown coefficients in the expansion were obtained by taking a finite number of terms in the series. In another study, Wong et al. [9] considered the two-dimensional response of a tunnel with a non-circular cross-section embedded in an elastic half-space by use of a technique involving wave function expansion in the half-space combined with a finite element representation of the tunnel and its vicinity. Balendra et al. [10] studied the in-plane vibrations of a tunnel-soil-building system in a viscoelastic half-space. The displacement fields were formulated by the method of wave function expansion and the boundary conditions were satisfied only at a finite number of points along the traction-free surface, the tunnel-soil interface and the soil-foundation interface. Thus, the unknown wave function coefficients were solved by a least squares approach. Lee and Karl [11] studied the scattering and diffraction of plane waves by underground, circular, cylindrical cavities at various depths in a half-space. In the study, they used the Fourier-Bessel series and approximated the half-space with a large diameter elastic cylinder.

Luco and Barros [12,13] studied the seismic response of a cylindrical shell embedded in a layered viscoelastic half-space by use of a technique that combines an indirect integral representation for the exterior domain with a simplified shell theory for the internal tunnel representation. Guan and Moore [14] studied the dynamic response of multiple cavities deeply buried in a viscoelastic medium that were subjected to moving or seismic loading by using the Fourier-Bessel series. In another study [15], they investigated the dynamic response of twin lined tunnels in an infinite medium subjected to seismic loadings using the method of successive reflection. They provided a comparison of the half-space and full-space results. Stamos and Beskos [16] studied the dynamic response of an infinitely long cylindrical tunnel buried in a half-space that was subjected to plane harmonic waves by using a special boundary element method in the frequency domain. Davis et al. [17] studied the transverse response of cylindrical cavities and pipes embedded in a half-space that were subjected to incident SV waves. The solution was obtained by using Fourier-Bessel series and a convex approximation of the half-space free surface. Considering the half-space to be a saturated poroelastic, Liang et al. [18] investigated the diffraction of incident plane SV waves by a circular cavity using a wave-function series and a downward concave approximation of the half-space free surface. More recently, Jiang et al. [19] studied the scattering of plane waves by a cylindrical cavity with lining in a poroelastic half-space by using the complex variable function method.

In this study, dynamic response of an elastic half-space with a cylindrical cavity of circular cross-section is analyzed. The cavity lies parallel to the plane-free surface of the medium at a finite depth and is subjected to a harmonic normal pressure at the inner surface. By introducing potentials, the governing equations are decoupled and reduced to Helmholtz equations satisfied by the potentials. The series solution for these equations is obtained via the wave function expansion method. The boundary conditions at the invert of the cavity are satisfied exactly, whereas they are satisfied only at a finite number of points along the traction-free surface of the half-space. Thus, the unknown wave function coefficients are solved by a least squares approach. Once the unknown coefficients are determined, the displacement and stress at any point in the medium can be calculated in a straightforward manner.

\section{Formulation of problem}

Consider a linearly elastic, homogeneous and isotropic half-space with a circular cavity of radius $a$. The cavity is considered to be infinitely long, lying parallel to the plane-free surface of the medium at a finite depth of $H$, and subjected to a uniformly distributed harmonic pressure $P(t)=P_{0} e^{-i \omega t}$ at the inner surface (Fig. 1). The axis of the cavity is taken to coincide with the $z$-axis. Given that the loading and the geometry of the cavity are assumed to be $z$-independent, the problem is two-dimensional along the $x$ and $y$ axes, and corresponds to the plane strain case. Thus, with the use of polar coordinates, the displacement vector $\mathbf{u}$ of a point will be a function of $r, \theta$ and $t$. The displacement vector $\mathbf{u}$ in the soil medium must satisfy the Navier equation in the absence of body forces

$$
(\lambda+\mu) \nabla \nabla \cdot \mathbf{u}+\mu \nabla^{2} \mathbf{u}+\rho \omega^{2} \mathbf{u}=0
$$

where $\lambda$ and $\mu$ are the Lamé constants, and $\rho$ is the mass density. It is assumed that the dependence on time is a simple harmonic of the form $e^{-i \omega t}$, where $i=\sqrt{-1}$. For brevity, the harmonic term $e^{-i \omega t}$ is henceforth suppressed from all expressions in the sequence. Using the Helmholtz separation theorem [3], the vector $\mathbf{u}$ can be written in 


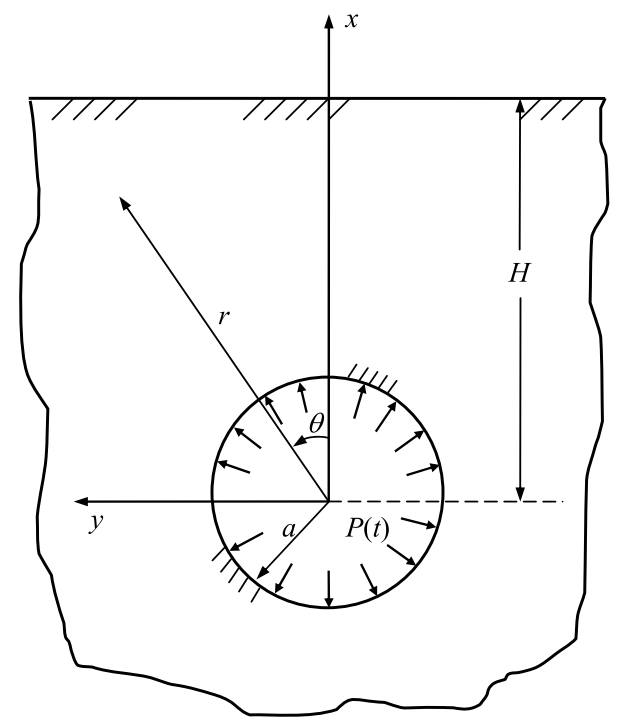

Fig. 1. Cylindrical cavity embedded in a half-space.

terms of a dilatational scalar potential $\varphi(r, \theta)$ and an equivoluminal vector potential $\psi(\mathrm{r}, \theta)=\psi(\mathrm{r}, \theta) \mathbf{e}_{z}$ with the form

$$
\mathbf{u}=\nabla \varphi+\nabla \times \boldsymbol{\psi} ; \nabla \cdot(\boldsymbol{\psi})=0
$$

where $\mathbf{e}_{z}$ is the unit vector along the $z$-axis. Eq. (2) is a solution of Eq. (1) if the displacement potentials are solutions of the following reduced wave equations for steady state two-dimensional wave propagation:

$$
\begin{aligned}
& \nabla^{2} \varphi+k_{1}^{2} \varphi=0 \\
& \nabla^{2} \psi+k_{2}^{2} \psi=0
\end{aligned}
$$

where $k_{1}=\omega \sqrt{\rho /(\lambda+2 \mu)}$ and $k_{2}=\omega \sqrt{\rho / \mu}$ are the longitudinal and shear wave numbers, respectively, $\omega$ is the circular frequency, and $\nabla^{2}$ is the two-dimensional Laplacian operator $\nabla^{2}=\left(\partial^{2} / \partial r^{2}\right)+(1 / r)(\partial / \partial r)+$ $\left(1 / r^{2}\right)\left(\partial^{2} / \partial \theta^{2}\right)$. The components of the displacement vector $\mathbf{u}$ in the radial direction $\mathbf{u}_{r}$ and circumferential direction $\mathbf{u}_{\theta}$ are given by $\mathbf{u}_{r}=u_{r} \mathbf{e}_{r}$ and $\mathbf{u}_{\theta}=u_{\theta} \mathbf{e}_{\theta}$. The unit vectors $\mathbf{e}_{r}$ and $\mathbf{e}_{\theta}$ are in the radial and circumferential directions, respectively. Hence $\mathbf{u}=\mathbf{u}_{r}+\mathbf{u}_{\theta}=u_{r} \mathbf{e}_{r}+u_{\theta} \mathbf{e}_{\theta}$ where the components of the displacement vector are given by

$$
\begin{aligned}
& u_{r}=\frac{\partial \varphi}{\partial r}+\frac{\partial \psi}{r \partial \theta} \\
& u_{\theta}=\frac{\partial \varphi}{r \partial \theta}-\frac{\partial \psi}{\partial r}
\end{aligned}
$$

Using Hook's law and the expressions for the components of the strain-tensor, the components of the associated stress field can be written as:

$$
\begin{aligned}
\tau_{r r} & =(\lambda+2 \mu) \frac{\partial u_{r}}{\partial r}+\frac{\lambda}{r}\left(u_{r}+\frac{\partial u_{\theta}}{\partial \theta}\right) \\
\tau_{\theta \theta} & =\lambda \frac{\partial u_{r}}{\partial r}+\frac{1}{r}(\lambda+2 \mu)\left(u_{r}+\frac{\partial u_{\theta}}{\partial \theta}\right) \\
\tau_{r \theta} & =\frac{\mu}{r}\left(r \frac{\partial u_{\theta}}{\partial r}-u_{\theta}+\frac{\partial u_{r}}{\partial \theta}\right)
\end{aligned}
$$


Using the method of separation of variables and noting that the number $n$ must be an integer for a periodic solution, the solutions of Eqs (3) and (4) can be written [2] as

$$
\begin{aligned}
& \varphi=\sum_{n=-\infty}^{\infty}\left[A_{n} J_{n}\left(k_{1} r\right)+B_{n} Y_{n}\left(k_{1} r\right)\right] e^{i n \theta} \\
& \psi=\sum_{n=-\infty}^{\infty}\left[C_{n} J_{n}\left(k_{2} r\right)+D_{n} Y_{n}\left(k_{2} r\right)\right] e^{i n \theta}
\end{aligned}
$$

where $J_{n}$ and $Y_{n}$ are Bessel functions of the first and second kind, order $n$, and $A_{n}, B_{n}, C_{n}$ and $D_{n}$ are arbitrary constants to be determined from the boundary conditions. Using the relations $J_{-n}=(-1)^{n} J_{n}$ and $Y_{-n}=(-1)^{n} Y_{n}$, and considering the potentials $\varphi$ and $\psi$ to be symmetric and antisymmetric, i.e., $\varphi(r, \theta)=\varphi(r,-\theta)$ and $\psi(r, \theta)=$ $-\psi(r,-\theta)$, Eqs (10) and (11) can be written in the form

$$
\begin{aligned}
& \varphi=\sum_{n=0}^{\infty}\left[A_{n} J_{n}\left(k_{1} r\right)+B_{n} Y_{n}\left(k_{1} r\right)\right] \cos n \theta \\
& \psi=\sum_{n=0}^{\infty}\left[C_{n} J_{n}\left(k_{2} r\right)+D_{n} Y_{n}\left(k_{2} r\right)\right] \sin n \theta
\end{aligned}
$$

Substituting Eqs (12) and (13) into Eqs (5) and (6), the displacement field is obtained as:

$$
\begin{aligned}
u_{r}= & \sum_{n=0}^{\infty}\left[A_{n}\left(\frac{n}{r} J_{n}\left(k_{1} r\right)-k_{1} J_{n+1}\left(k_{1} r\right)\right)+B_{n}\left(\frac{n}{r} Y_{n}\left(k_{1} r\right)-k_{1} Y_{n+1}\left(k_{1} r\right)\right)\right. \\
& \left.+C_{n} \frac{n}{r} J_{n}\left(k_{2} r\right)+D_{n} \frac{n}{r} Y_{n}\left(k_{2} r\right)\right] \cos n \theta \\
u_{\theta}= & \sum_{n=0}^{\infty}\left[-A_{n} \frac{n}{r} J_{n}\left(k_{1} r\right)-B_{n} \frac{n}{r} Y_{n}\left(k_{1} r\right)-C_{n}\left(\frac{n}{r} J_{n}\left(k_{2} r\right)-k_{2} J_{n+1}\left(k_{2} r\right)\right)\right. \\
& \left.-D_{n}\left(\frac{n}{r} Y_{n}\left(k_{2} r\right)-k_{2} Y_{n+1}\left(k_{2} r\right)\right)\right] \sin n \theta
\end{aligned}
$$

Likewise, substituting Eqs (14) and (15) into Eqs (7-9), the associated stress field can be written as:

$$
\begin{aligned}
\tau_{r r}= & \frac{2 \mu}{r^{2}} \sum_{n=0}^{\infty}\left\{A_{n}\left[\beta_{1} J_{n}\left(k_{1} r\right)+k_{1} r J_{n+1}\left(k_{1} r\right)\right]+B_{n}\left[\beta_{1} Y_{n}\left(k_{1} r\right)+k_{1} r Y_{n+1}\left(k_{1} r\right)\right]\right. \\
& \left.+C_{n}\left[\beta_{2} J_{n}\left(k_{2} r\right)-n k_{2} r J_{n+1}\left(k_{2} r\right)\right]+D_{n}\left[\beta_{2} Y_{n}\left(k_{2} r\right)-n k_{2} r Y_{n+1}\left(k_{2} r\right)\right]\right\} \cos n \theta \\
\tau_{\theta \theta}= & \frac{2 \mu}{r^{2}} \sum_{n=0}^{\infty}\left\{A_{n}\left[\beta_{3} J_{n}\left(k_{1} r\right)-k_{1} r J_{n+1}\left(k_{1} r\right)\right]+B_{n}\left[\beta_{3} Y_{n}\left(k_{1} r\right)-k_{1} r Y_{n+1}\left(k_{1} r\right)\right]\right. \\
& \left.+C_{n}\left[-\beta_{2} J_{n}\left(k_{2} r\right)+n k_{2} r J_{n+1}\left(k_{2} r\right)\right]+D_{n}\left[-\beta_{2} Y_{n}\left(k_{2} r\right)+n k_{2} r Y_{n+1}\left(k_{2} r\right)\right]\right\} \cos n \theta \\
\tau_{r \theta}= & \frac{2 \mu}{r^{2}} \sum_{n=0}^{\infty}\left\{A_{n}\left[-\beta_{2} J_{n}\left(k_{1} r\right)+n k_{1} r J_{n+1}\left(k_{1} r\right)\right]+B_{n}\left[-\beta_{2} Y_{n}\left(k_{1} r\right)+n k_{1} r Y_{n+1}\left(k_{1} r\right)\right]\right. \\
& \left.+C_{n}\left[-\beta_{1} J_{n}\left(k_{2} r\right)-k_{2} r J_{n+1}\left(k_{2} r\right)\right]+D_{n}\left[-\beta_{1} Y_{n}\left(k_{2} r\right)-k_{2} r Y_{n+1}\left(k_{2} r\right)\right]\right\} \sin n \theta
\end{aligned}
$$

where

$$
\beta_{1}=n^{2}-n-0.5 k_{2}^{2} r^{2}, \quad \beta_{2}=n^{2}-n, \quad \beta_{3}=-n^{2}+n+k_{1}^{2} r^{2}-0.5 k_{2}^{2} r^{2} .
$$


The boundary conditions associated with the problem will be written at the invert of the cavity and at the free surface of the half-space. Given that the cavity is subjected to an internal pressure of $P_{0}$ in magnitude at $r=a$, the boundary conditions at the cavity surface are:

$$
\begin{aligned}
& \tau_{r r}=-P_{0} \\
& \tau_{r \theta}=0
\end{aligned}
$$

The zero-stress boundary conditions at the free surface of the half-space, at $x=H$, can be expressed as:

$$
\begin{aligned}
& \tau_{x x}=0 \\
& \tau_{x y}=0
\end{aligned}
$$

where $\tau_{i j}$ is the stress tensor in Cartesian coordinates. To use the conditions given by Eqs (20a) and (20b), the stress field, which is expressed in polar coordinates, must be transformed into Cartesian coordinates with the relations

$$
\begin{aligned}
& \tau_{x x}=\tau_{r r} \cos ^{2} \theta+\tau_{\theta \theta} \sin ^{2} \theta+2 \tau_{r \theta} \sin \theta \cos \theta \\
& \tau_{x y}=-\left(\tau_{r r}-\tau_{\theta \theta}\right) \sin \theta \cos \theta+\tau_{r \theta}\left(\cos ^{2} \theta-\sin ^{2} \theta\right) .
\end{aligned}
$$

Similarly, the displacement components in Cartesian coordinates, which will be used to show the displacement distribution at the free-surface, are given by

$$
u_{x}=u_{r} \cos \theta-u_{\theta} \sin \theta ; \quad u_{y}=u_{r} \sin \theta+u_{\theta} \cos \theta
$$

Substituting Eqs (16-18) into Eq. (21) and arranging the terms appropriately, the transformed stress components are obtained as:

$$
\begin{aligned}
& \tau_{x x}=\frac{2 \mu}{r^{2}} \sum_{n=0}^{\infty}\left\langle A_{n}\left\{\begin{array}{l}
{\left[S_{1} J_{n}\left(k_{1} r\right)+\left(S_{2} J_{n}\left(k_{1} r\right)+k_{1} r J_{n+1}\left(k_{1} r\right)\right) \cos 2 \theta\right] \cos n \theta} \\
+\left[S_{3} J_{n}\left(k_{1} r\right)+n k_{1} r J_{n+1}\left(k_{1} r\right)\right] \sin 2 \theta \sin n \theta
\end{array}\right\}\right. \\
& +B_{n}\left\{\begin{array}{l}
{\left[S_{1} Y_{n}\left(k_{1} r\right)+\left(S_{2} Y_{n}\left(k_{1} r\right)+k_{1} r Y_{n+1}\left(k_{1} r\right)\right) \cos 2 \theta\right] \cos n \theta} \\
+\left[S_{3} Y_{n}\left(k_{1} r\right)+n k_{1} r Y_{n+1}\left(k_{1} r\right)\right] \sin 2 \theta \sin n \theta
\end{array}\right\} \\
& +C_{n}\left\{\left[-S_{3} J_{n}\left(k_{2} r\right)-n k_{2} r J_{n+1}\left(k_{2} r\right)\right] \cos 2 \theta \cos n \theta+\left[S_{4} J_{n}\left(k_{2} r\right)-k_{2} r J_{n+1}\left(k_{2} r\right)\right] \sin 2 \theta \sin n \theta\right\} \\
& \left.+D_{n}\left\{\left[-S_{3} Y_{n}\left(k_{2} r\right)-n k_{2} r Y_{n+1}\left(k_{2} r\right)\right] \cos 2 \theta \cos n \theta+\left[S_{4} Y_{n}\left(k_{2} r\right)-k_{2} r Y_{n+1}\left(k_{2} r\right)\right] \sin 2 \theta \sin n \theta\right\}\right\rangle \\
& \tau_{x y}=\frac{2 \mu}{r^{2}} \sum_{n=0}^{\infty}\left\langle A_{n}\left\{\begin{array}{l}
{\left[S_{2} J_{n}\left(k_{1} r\right)+k_{1} r J_{n+1}\left(k_{1} r\right)\right] \sin 2 \theta \cos n \theta} \\
-\left[S_{3} J_{n}\left(k_{1} r\right)+n k_{1} r J_{n+1}\left(k_{1} r\right)\right] \cos 2 \theta \sin n \theta
\end{array}\right\}\right. \\
& +B_{n}\left\{\left[S_{2} Y_{n}\left(k_{1} r\right)+k_{1} r Y_{n+1}\left(k_{1} r\right)\right] \sin 2 \theta \cos n \theta-\left[S_{3} Y_{n}\left(k_{1} r\right)+n k_{1} r Y_{n+1}\left(k_{1} r\right)\right] \cos 2 \theta \sin n \theta\right\} \\
& +C_{n}\left\{\left[-S_{3} J_{n}\left(k_{2} r\right)-n k_{2} r J_{n+1}\left(k_{2} r\right)\right] \sin 2 \theta \cos n \theta-\left[S_{4} J_{n}\left(k_{2} r\right)-k_{2} r J_{n+1}\left(k_{2} r\right)\right] \cos 2 \theta \sin n \theta\right\} \\
& \left.+D_{n}\left\{\left[-S_{3} Y_{n}\left(k_{2} r\right)-n k_{2} r Y_{n+1}\left(k_{2} r\right)\right] \sin 2 \theta \cos n \theta-\left[S_{4} Y_{n}\left(k_{2} r\right)-k_{2} r Y_{n+1}\left(k_{2} r\right)\right] \cos 2 \theta \sin n \theta\right\}\right\rangle
\end{aligned}
$$

where

$$
S_{1}=0.5\left(k_{1}^{2} r^{2}-k_{2}^{2} r^{2}\right), \quad S_{2}=n^{2}-n-0.5 k_{1}^{2} r^{2}, \quad S_{3}=-n^{2}+n, \quad S_{4}=-n^{2}+n+0.5 k_{2}^{2} r^{2} .
$$

Because of the difficulty in taking into account an infinite number of terms in the series given above, especially for the functions $Y_{n}$ for a small argument with a large index, the series are truncated at a finite number $N$. In this case, the number of unknown constants $\left(A_{n}, B_{n}, C_{n}, D_{n}, n=0,1,2, \ldots, N\right)$ in the equations becomes $4 \times N+2$, in which $C_{0}=D_{0}=0$ for $n=0$ in Eq. (16). In order to obtain these constants, the boundary conditions given by (19a-20b) will be used. Substituting Eq. (16) into Eq. (19a) and considering that the solution must be valid for all $\theta$, $N+1$ algebraic equations are obtained. This solution is accomplished by equating the coefficient of $\cos n \theta$ to $P_{0}$ for $n=0$ and zero for $n>0$, respectively. Similarly, substituting Eq. (18) into Eq. (19b) and equating the coefficient of $\sin n \theta$ to zero for $n>0, N$ equations are obtained. Thus, with the use of the boundary conditions at $r=a$, 
$2 \times N+1$ algebraic equations are obtained. The necessary $2 \times N+1$ equations will be obtained from the boundary conditions (20a) and (20b), which require the stresses at each point of the free surface to equal zero. Since there are an infinite number of points along the surface, the use of these conditions leads to an infinite set of algebraic equations. Besides, these boundary conditions cannot be satisfied exactly at each point as the series are truncated by keeping only a finite number of terms in the summations. Therefore, the boundary conditions (20a) and (20b) are satisfied approximately only at a finite number of points along the free surface by applying the least-squares technique. For this purpose, $2 \times N+1$ constants are solved in terms of other constants from the $2 \times N+1$ equations obtained above, i.e., $A_{0}=f\left(B_{0}\right), A_{n}=f\left(C_{n}, D_{n}\right)$ and $B_{n}=f\left(C_{n}, D_{n}\right)$, and substituted into Eqs (23) and (24). Thus, the number of constants in these equations reduces to $2 \times N+1$. Subsequently, these $2 \times N+1$ constants can be obtained from a set of algebraic equations that will be established by taking $r_{k}=H / \cos \theta_{k}$ in Eqs (23) and (24) and using the least-squares technique given by

$$
\Pi=\sum_{k=1}^{M}\left[\left(\tau_{x x}^{(k)}-\hat{\tau}_{x x}^{(k)}\right)^{2}+\left(\tau_{x y}^{(k)}-\hat{\tau}_{x y}^{(k)}\right)^{2}\right]=\min
$$

Here, $M$ is the number of points on the surface, $\tau_{x x}^{(k)}$ and $\tau_{x y}^{(k)}$ are the stress components to be computed approximately at any point $k$, and $\hat{\tau}_{x x}^{(k)}$ and $\hat{\tau}_{x y}^{(k)}$ are the external stress components at these points. With the use of Eq. (25), a set of algebraic equations for the unknowns $B_{0}, C_{n}$ and $D_{n}$ can be found from:

$$
\begin{aligned}
& \frac{\partial \Pi}{\partial B_{0}}=\sum_{k=1}^{M}\left[\tau_{x x}^{(k)} \frac{\partial \tau_{x x}^{(k)}}{\partial B_{0}}+\tau_{x y}^{(k)} \frac{\partial \tau_{x y}^{(k)}}{\partial B_{0}}\right]=0 \\
& \frac{\partial \Pi}{\partial C_{n}}=\sum_{k=1}^{M}\left[\tau_{x x}^{(k)} \frac{\partial \tau_{x x}^{(k)}}{\partial C_{n}}+\tau_{x y}^{(k)} \frac{\partial \tau_{x y}^{(k)}}{\partial C_{n}}\right]=0, \quad n=1,2, \ldots, N \\
& \frac{\partial \Pi}{\partial D_{n}}=\sum_{k=1}^{M}\left[\tau_{x x}^{(k)} \frac{\partial \tau_{x x}^{(k)}}{\partial D_{n}}+\tau_{x y}^{(k)} \frac{\partial \tau_{x y}^{(k)}}{\partial D_{n}}\right]=0, \quad n=1,2, \ldots, N
\end{aligned}
$$

in which it is assumed that $\hat{\tau}_{x x}^{(k)}=\hat{\tau}_{x y}^{(k)}=0$ at $r_{k}=H / \cos \theta_{k}$ as the surface is traction-free. These $2 \times N+1$ equations are used in the determination of the $2 \times N+1$ constants. After the determination of these constants, the other $2 \times N+1$ constants $\left(A_{0}, A_{n}, B_{n}\right)$ are obtained from the relations $A_{0}=f\left(B_{0}\right), A_{n}=f\left(C_{n}, D_{n}\right)$ and $B_{n}=f\left(C_{n}, D_{n}\right)$. Then, with the use of these constants in the solutions, the displacement and stress components at any point can be calculated. The numerical results, which will be presented in the next section, were obtained with the use of non-dimensional spatial coordinates and non-dimensional cavity depth in the displacement and stress field quantities. For this purpose, the spatial coordinates and the cavity depth were non-dimensionalized with respect to radius $a(\bar{x}=x / a, \bar{y}=y / a, \bar{r}=r / a$ and $\bar{H}=H / a)$ and parameters $\bar{k}_{1}=k_{1} a$ and $\bar{k}_{2}=k_{2} a$ were introduced in the displacement potentials $\varphi$ and $\psi$. The overbars were then dropped in the evaluation of all field quantities.

\section{Numerical results and discussion}

As indicated in the foregoing, the least-squares technique is used for the boundary conditions on the free surface of the half-space. This technique requires the use of numerical values for satisfying the boundary conditions at the points along the surface; thus, modal behavior cannot be observed in the system. In contrast, a simple harmonic distributed force with frequency $\omega$ is applied at the invert of the cavity, which causes forced motion in the system; therefore, the response of the system is dependent on the forcing frequency because the material properties of the medium are known. The dependence on the frequency is determined by the well-known relations $k_{1}=\omega / c_{1}$ and $k_{2}=\omega / c_{2}$, where $k_{1}$ and $k_{2}$ are longitudinal and shear wave numbers and $c_{1}=\sqrt{(\lambda+2 \mu) / \rho}$ and $c_{2}=\sqrt{\mu / \rho}$ are the longitudinal and shear wave speeds, respectively. Using these relations, the wave numbers $\left(k_{1}, k_{2}\right)$ are obtained numerically for a given forcing frequency and are then used to represent the behavior of the system. Moreover, it 


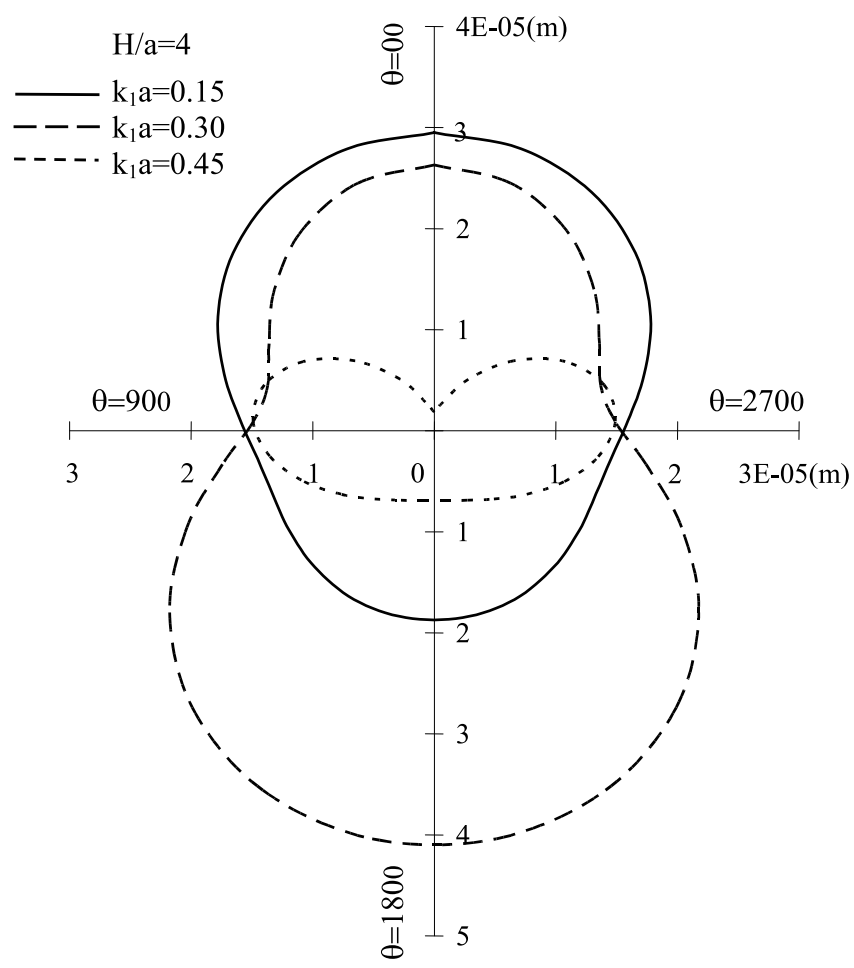

Fig. 2. Variation of the radial displacement $\left(u_{r}\right)$ with $\theta$ at $r=a$ for various values of wave number $k_{1} a$.

should be noted that a dispersion relation, which is encountered in typical wave propagation problems, cannot be obtained here because not all of the boundary conditions are homogeneous; additionally, some of them are treated numerically at the free surface (i.e., they are approximately satisfied for a finite region by considering a finite number of terms in the series solutions).

The numerical computations were carried out for a rock-like medium with properties $\rho=2665 \mathrm{~kg} / \mathrm{m}^{3}, E=$ $7.567 \times 10^{9} \mathrm{~N} / \mathrm{m}^{2}$ and $\nu=0.25[8]$. The internal pressure $P_{0}$ on the cavity surface, the number $N$, and the region at the free surface were taken to be $P_{0}=0.1 \mathrm{MPa}, N=8$ and $-10 \times H \leqslant y \leqslant 10 \times H$, respectively. The non-dimensional wave number $\left(k_{1} a\right)$ and the non-dimensional depth $(H / a)$ were used to represent the behavior. The figures, in which the displacement and stress distributions are shown for the cavity surface, were depicted with respect to the absolute values. Due to the symmetry of the system with respect to the $x$-axis, the displacement and stress distributions become symmetrical about this axis in all figures.

Figures 2-4 show the effect of varying the wave number on the radial displacement, circumferential displacement and the circumferential normal stress at the cavity surface $r=a$ for a fixed depth $H / a=4$. Figure 2 shows that radial displacement in the upper half of the cavity decreases as $k_{1} a$ increases. However, in the lower half of the cavity, radial displacement initially increases as $k_{1} a$ increases and then decreases as $k_{1} a$ increases further. Also, it is found that the maximum values, which depend on the wave number, occur at $\theta=0^{\circ}$ and $\theta=180^{\circ}$, i.e., at the nearest and farthest points from the free surface. The distribution of circumferential displacement is shown in Fig. 3. Depending on the $k_{1} a$ values, there are two local maxima in the lower and upper half of the cavity. As for the radial displacement, maximum circumferential displacements occur in the lower half of the cavity. Comparisons of the displacements in Figs 2 and 3 show that the radial displacements are larger than the circumferential displacements. Figure 4 shows the distribution of the circumferential normal stress along the cavity surface. As for the circumferential displacement, the circumferential normal stress initially increases as $k_{1} a$ increases and then decreases as $k_{1} a$ increases further. The circumferential normal stress is found to be nearly symmetric about the horizontal ( $y$ ) axis at small values of $k_{1} a$, with maxima occurring at $\theta=90^{\circ}$ and $\theta=270^{\circ}$.

Figure 5 shows the variation of the free-surface vertical displacements $\left(u_{x}\right)$ with respect to the horizontal coordinate $(y / a)$ for different values of $k_{1} a$ at a fixed depth $H / a=4$. It can be seen that vertical displacements decrease as $y / a$ 


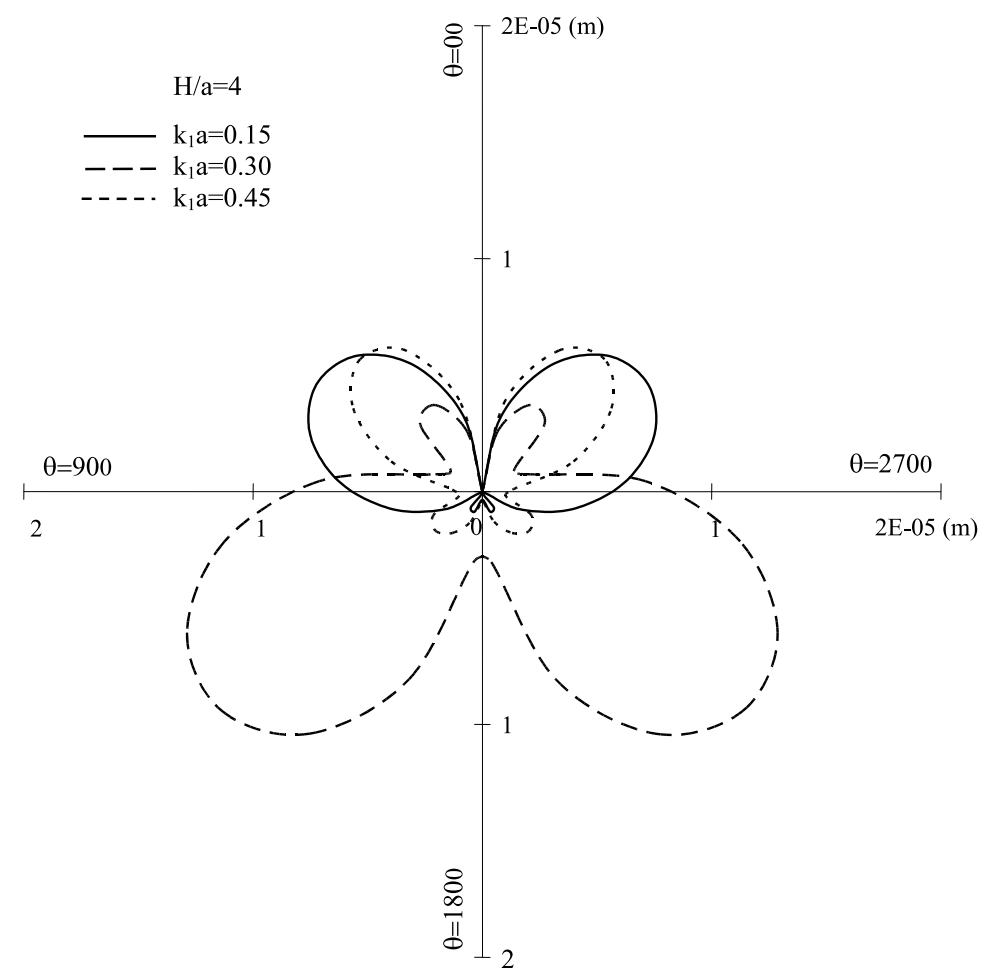

Fig. 3. Variation of the circumferential displacement $\left(u_{\theta}\right)$ with $\theta$ at $r=a$ for various values of wave number $k_{1} a$.

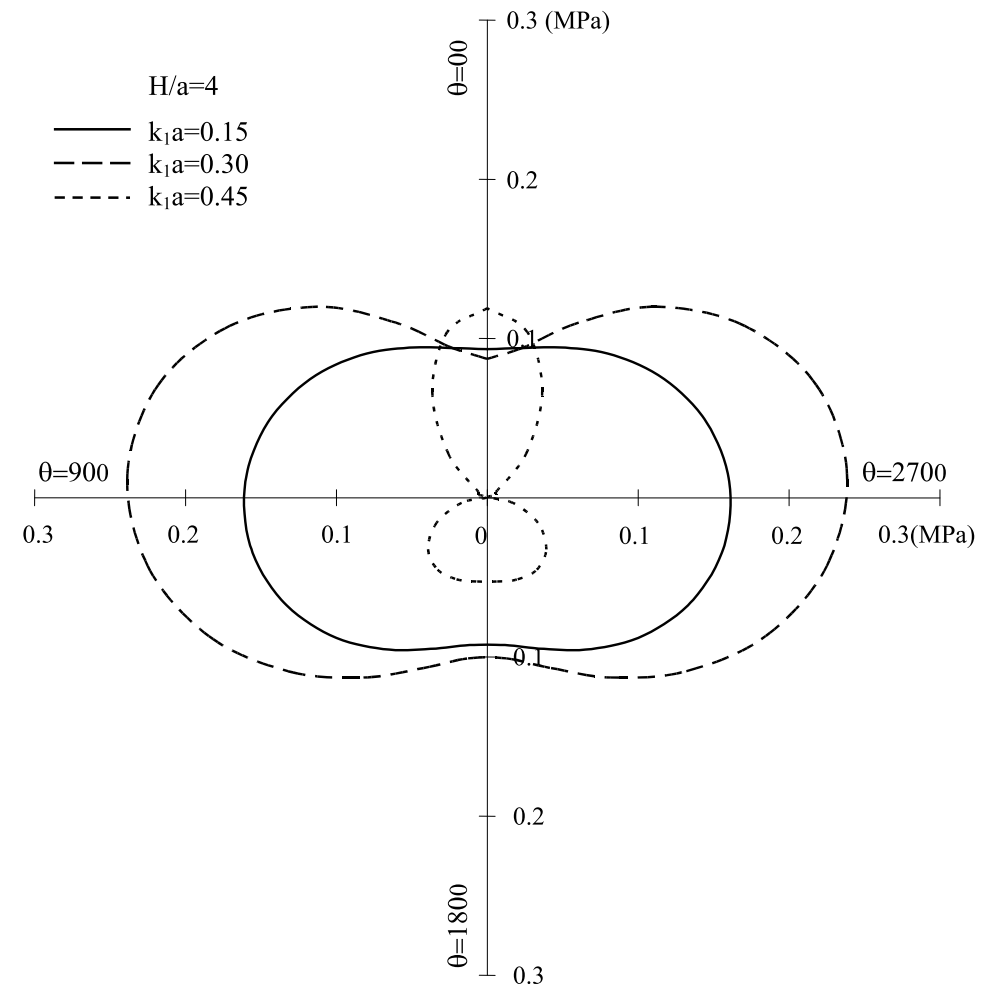

Fig. 4. Variation of the circumferential normal stress $\left(\tau_{\theta \theta}\right)$ with $\theta$ at $r=a$ for various values of wave number $k_{1} a$. 


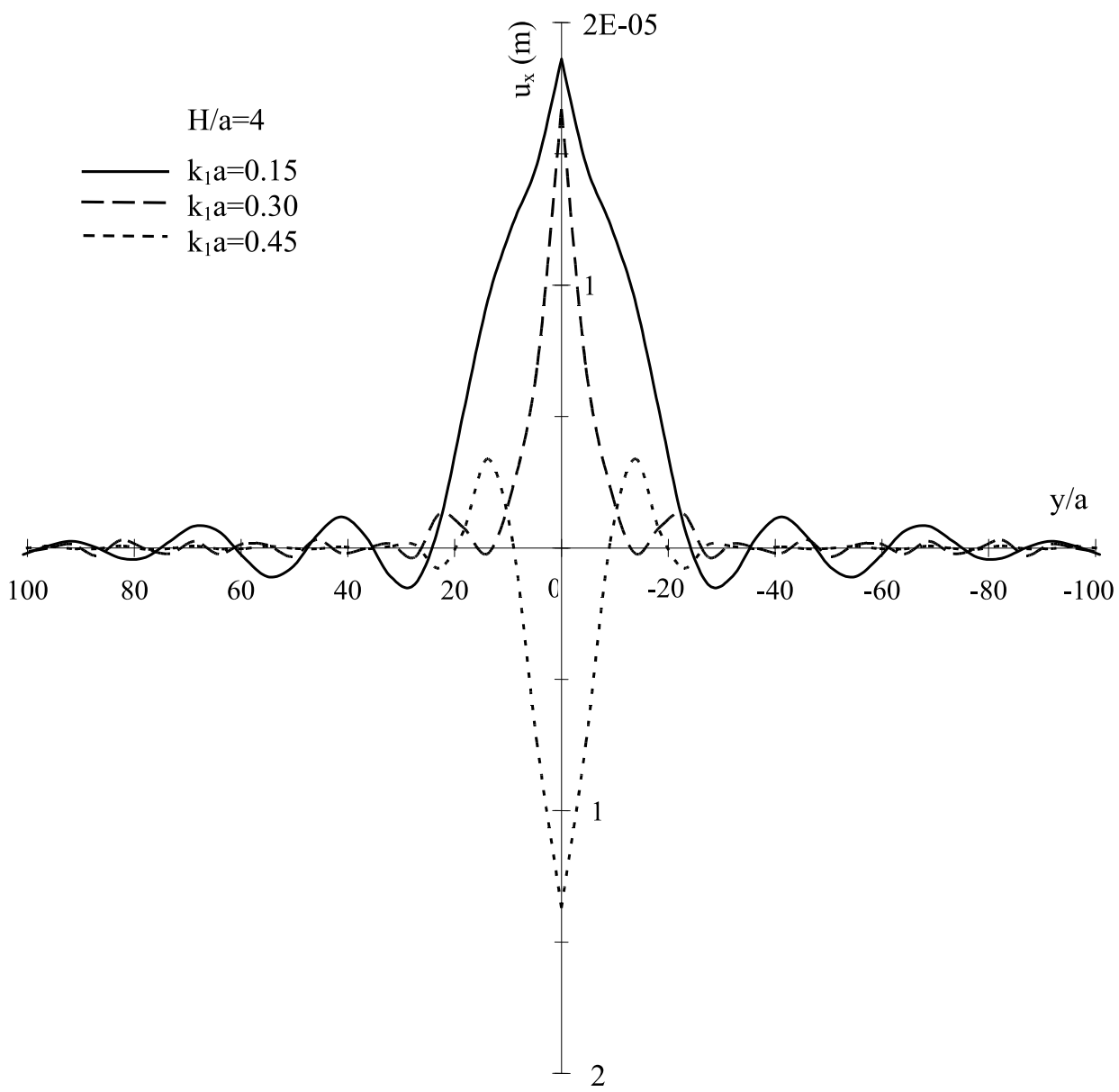

Fig. 5. Variation of the vertical displacement $\left(u_{x}\right)$ with $y / a$ at the free surface of the half-space for various values of wave number $k_{1} a$.

increases for all values of $k_{1} a$. Maximum displacements occurred at $y / a=0, x=H / a$, i.e., at the point nearest the top of the cavity, and their values decrease as $k_{1} a$ increases. It should be noted that the horizontal displacements (not shown here) are zero at this point due to symmetry about the vertical $(x)$ axis, and that the horizontal displacement values are small compared with the vertical displacements at other points of the free surface.

Figures 6-8 show the effect of varying the cavity depth $H / a$ on the radial displacement, circumferential displacement and the circumferential normal stress at the cavity surface $r=a$ for a fixed wave number $k_{1} a=0.3$. Figure 6 shows that the maximum radial displacement occurs at depth $H / a=4$, where the cavity is neither far away from the free surface nor very close to it. Also, it is observed that the radial displacement is nearly symmetric about the cavity center for $H / a=10$. With further increases in the cavity depth (not shown here), the contribution becomes more and more symmetric about the cavity center, which is consistent with the full-space solution. The distribution of circumferential displacement is shown in Fig. 7. It can be seen that increasing the cavity depth results in lower displacements at the cavity surface. Maximum values occur for the case $H / a=2$, i.e., when the cavity is nearest to the free surface. The distribution of circumferential normal stress is shown in Fig. 8. It can be seen that maximum values occurred at $\theta=90^{\circ}$ and $\theta=270^{\circ}$. As in the case of radial displacement, circumferential normal stress distribution is found to be nearly symmetric about the center of the cavity for $H / a=10$.

Figure 9 shows the variation of the free-surface vertical displacements $\left(u_{x}\right)$ with respect to the horizontal coordinate ( $y / a$ ) for different values of $H / a$ at a fixed wave number $k_{1} a=0.3$. It is seen that the vertical displacements decrease as $y / a$ increases for all cavity depths, and that the vertical displacement values at the points far away from the cavity become small compared with the respective values around the cavity. Maximum displacements occurred at $y / a=0$, 


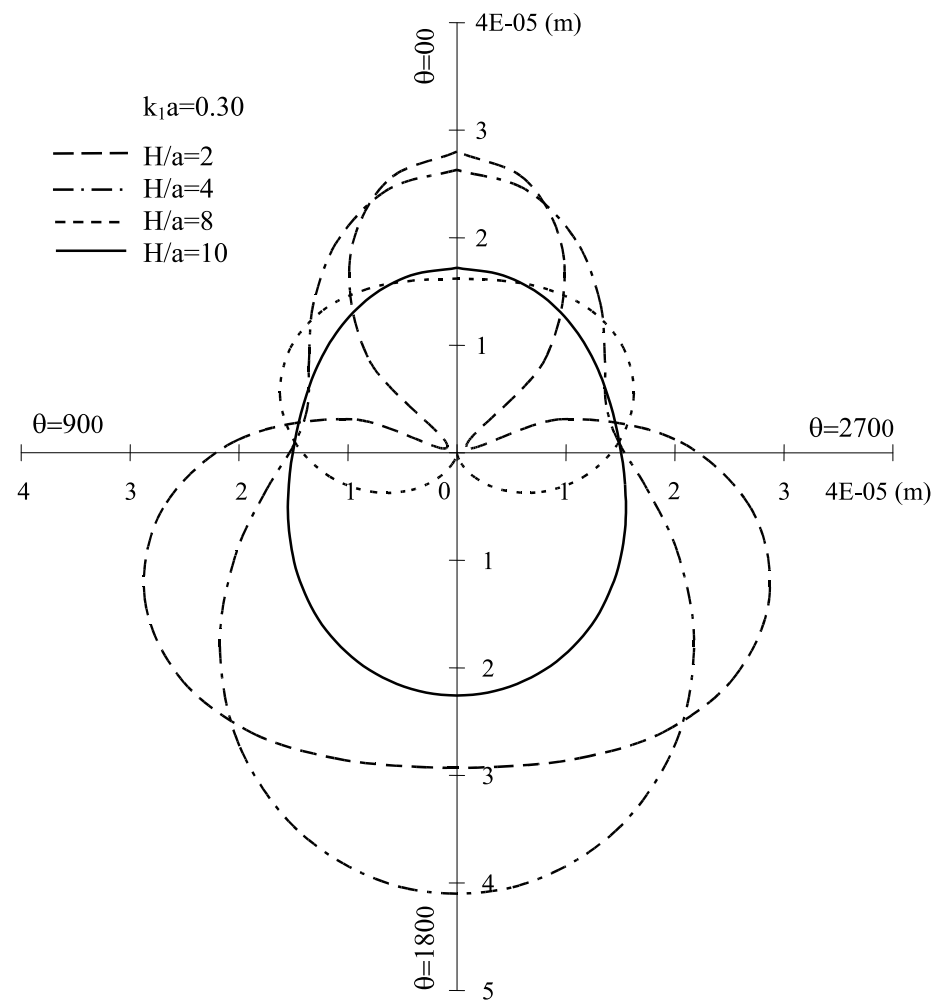

Fig. 6. Variation of the radial displacement $\left(u_{r}\right)$ with $\theta$ at $r=a$ for various values of depth $H / a$.

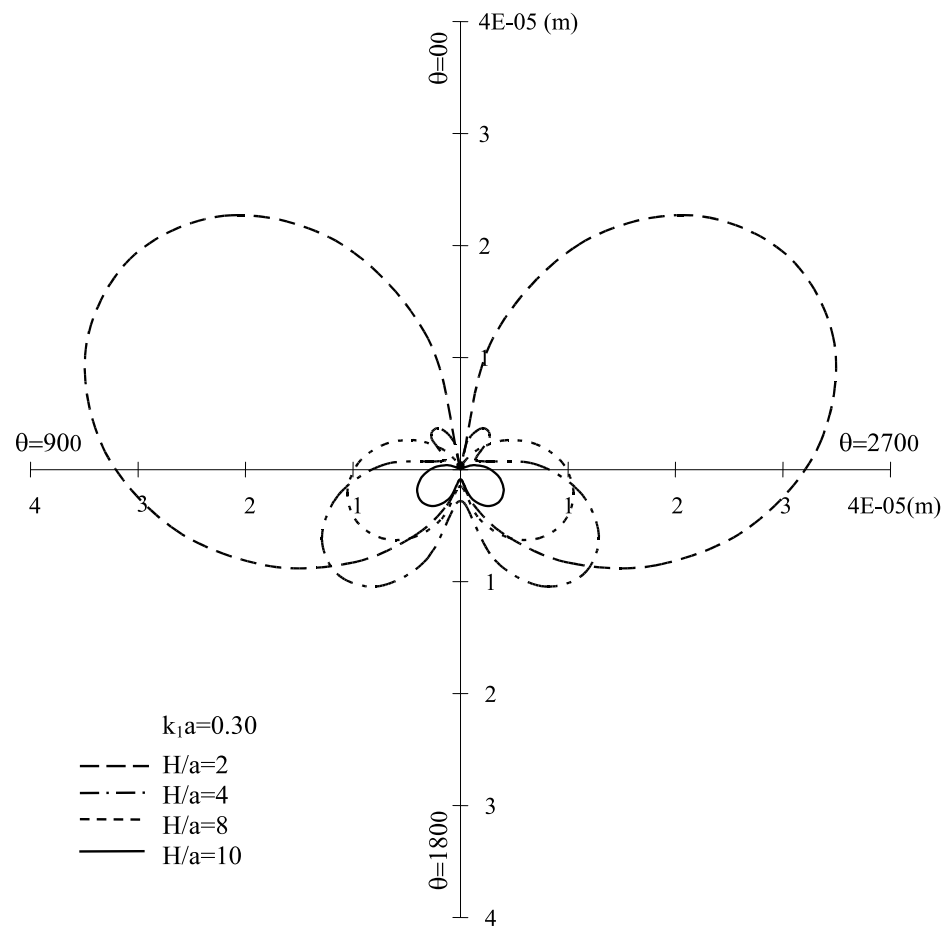

Fig. 7. Variation of the circumferential displacement $\left(u_{\theta}\right)$ with $\theta$ at $r=a$ for various values of depth $H / a$. 


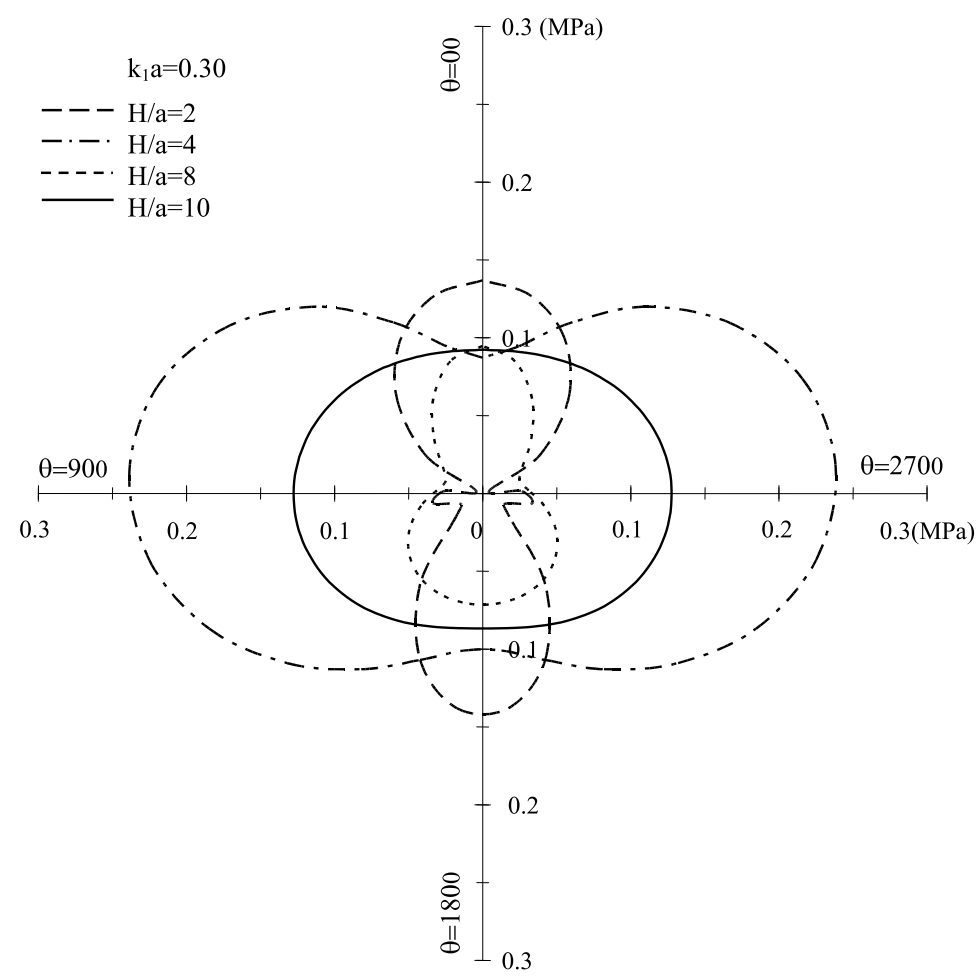

Fig. 8. Variation of the circumferential normal stress $\left(\tau_{\theta \theta}\right)$ with $\theta$ at $r=a$ for various values of depth $H / a$.

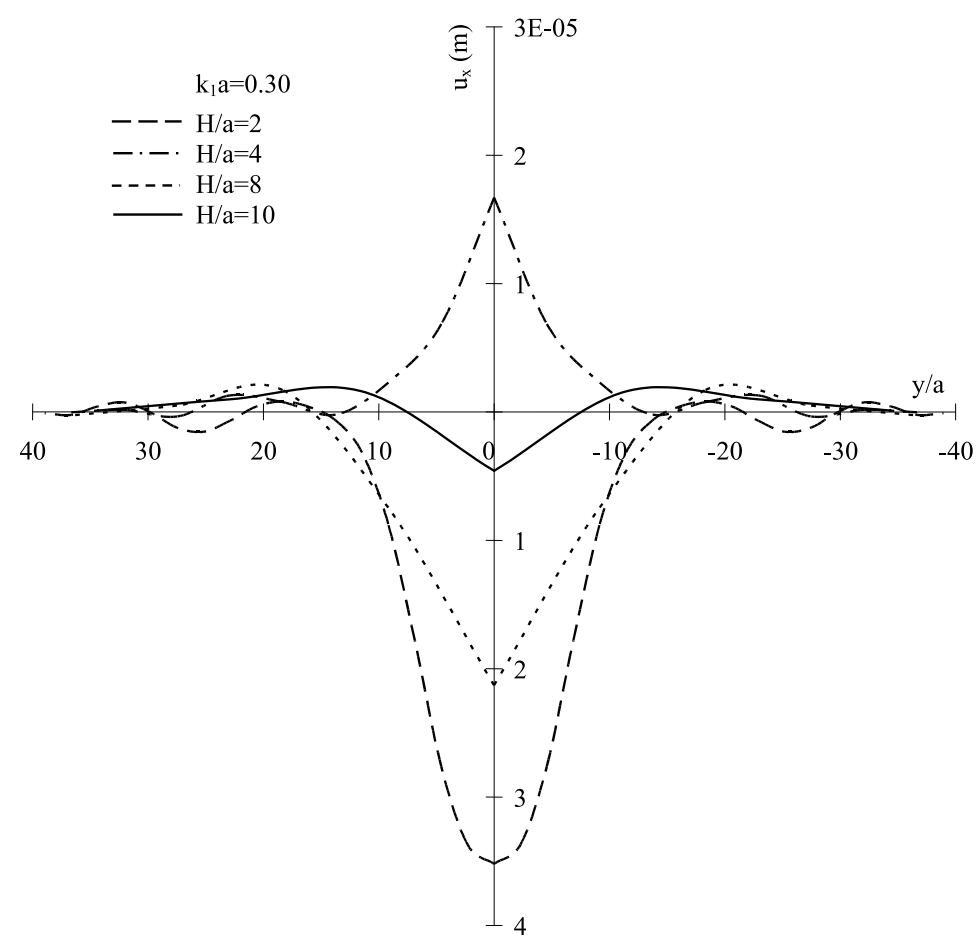

Fig. 9. Variation of the vertical displacement $\left(u_{x}\right)$ with $y / a$ at the free surface of the half-space for various values of depth $H / a$. 
$x=H / a$, i.e., at the point nearest to the top of the cavity, and the displacement values decreased significantly as the cavity depth $H / a$ increased.

\section{Conclusion}

The dynamic response of a half-space with a cylindrical cavity subjected to a uniform harmonic pressure at the inner surface has been studied. The effects of the wave number and depth on the displacement and stress distributions at the cavity surface, and at the free surface of the half-space have been presented in the figures. In general, it is found that maximum radial displacements at the cavity surface occurred at the top and bottom points, i.e., at the nearest and farthest points from the free surface of the half-space. With the increase of wave number and cavity depth, these values decrease at the top of the cavity, whereas they increase first and then decrease at the bottom of the cavity. Thus, it is concluded that the wave number and cavity depth have similar effects on the radial displacements. For a fixed wave number, the circumferential displacements on the cavity surface are found to decrease as the depth of the cavity increases. Maximum displacement is found to occur when the cavity is nearest the free surface. Maximum circumferential normal stresses at the cavity surface are found to occur on the horizontal and vertical axes depending on the wave number and depth. Maximum vertical displacements at the free surface of the half-space are found to occur at the point nearest to the cavity. These values, in absolute terms, decrease as the wave number and the depth of the cavity increase. It is concluded that both the displacements and stresses at the cavity surface, and the displacements at free surface of the half-space are significantly influenced by the wave number (i.e., the frequency) and the proximity of the cavity to the free surface.

\section{References}

[1] J.D. Achenbach, Wave propagation in elastic solids, North-Holland Publishing Co., Amsterdam, 1973.

[2] A.C. Eringen and E.S. Şuhubi, Elastodynamics, Vol. II, Academic Press, New-York, 1975.

[3] K.F. Graff, Wave motion in elastic solids, Clarendon Press, Oxford, 1975.

[4] Y.H. Pao and C.C. Mow, Diffraction of Elastic Waves and Dynamic Stress Concentrations, Crane, Russak\&Company, New-York, 1973.

[5] V.R.Thiruvenkatachar and K. Viswanathan, Dynamic response of an elastic half- space with cylindrical cavity to time-dependent surface tractions over the boundary of the cavity, Journal of Mathematics and Mechanics 14 (1965), 541-571.

[6] N. El-Akily and S.K. Datta, Response of a circular cylindrical shell to disturbances in a half-space, Earthquake Engineering and Structural Dynamics 8 (1980), 469-477.

[7] N. El-Akily and S.K. Datta, Response of a circular cylindrical shell to disturbances in a half-space.-numerical results, Earthquake Engineering and Structural Dynamics 9 (1981), 477-487.

[8] S.K. Datta, A.H. Shah and K.C. Wong, Dynamic stresses and displacements in buried pipe, ASCE Journal of Engineerig Mechanics 110(10) (1984), 1451-1466.

[9] K.C. Wong, A.H. Shah and S.K. Datta, Dynamic stresses and displacements in a buried tunnel, ASCE Journal of Engineerig Mechanics 111(2) (1985), 218-234.

[10] T. Balendra, C.G. Koh and Y.C. Ho, Dynamic response of buildings due to trains in underground tunnels, Earthquake Engineering and Structural Dynamics 20 (1991), 275-291.

[11] V.W. Lee and J. Karl, Diffraction of SV waves by underground, circular, cylindrical cavities, Soil Dynamics and Earthquake Engineering 11 (1992), 445-456.

[12] J.E. Luco and F.C.P. De Barros, Seismic response of a cylindrical shell embedded in a layered viscoelastic half-space. I: Formulation, Earthquake Engineering and Structural Dynamics 23 (1994), 553-567.

[13] J.E. Luco and F.C.P. De Barros, Seismic response of a cylindrical shell embedded in a layered viscoelastic half-space. II: Validation and numerical results, Earthquake Engineering and Structural Dynamics 23 (1994), 569-580.

[14] F. Guan and I.D. Moore, Three-dimensional dynamic response of twin cavities due to traveling loads, ASCE Journal of Engineerig Mechanics 120(3) (1994), 637-651.

[15] I.D. Moore and F. Guan, Three-dimensional dynamic response of lined tunnels due to incident seismic waves, Earthquake Engineering and Structural Dynamics 25 (1996), 357-369.

[16] A.A. Stamos and D.E. Beskos, 3-D seismic response analysis of long lined tunnels in half- Space, Soil Dynamics and Earthquake Engineering 15 (1996), 111-118.

[17] C.A. Davis, V.W. Lee and J.P. Bardet, Transverse response of underground cavities and pipes to incident SV waves, Earthquake Engineering and Structural Dynamics 30 (2001), 383-410.

[18] J. Liang, Z. Ba and V.W. Lee, Diffraction of plane SV waves by an underground circular cavity in a saturated poroelastic half-space, ISET Journal of Earthquake Technology 44(2) (2007), 341-375.

[19] L.F. Jiang, X.L. Zhou and J.H. Wang, Scattering of a plane wave by a lined cylindrical cavity in a poroelastic half-plane, Computers and Geotechnics 36 (2009), 773-786. 

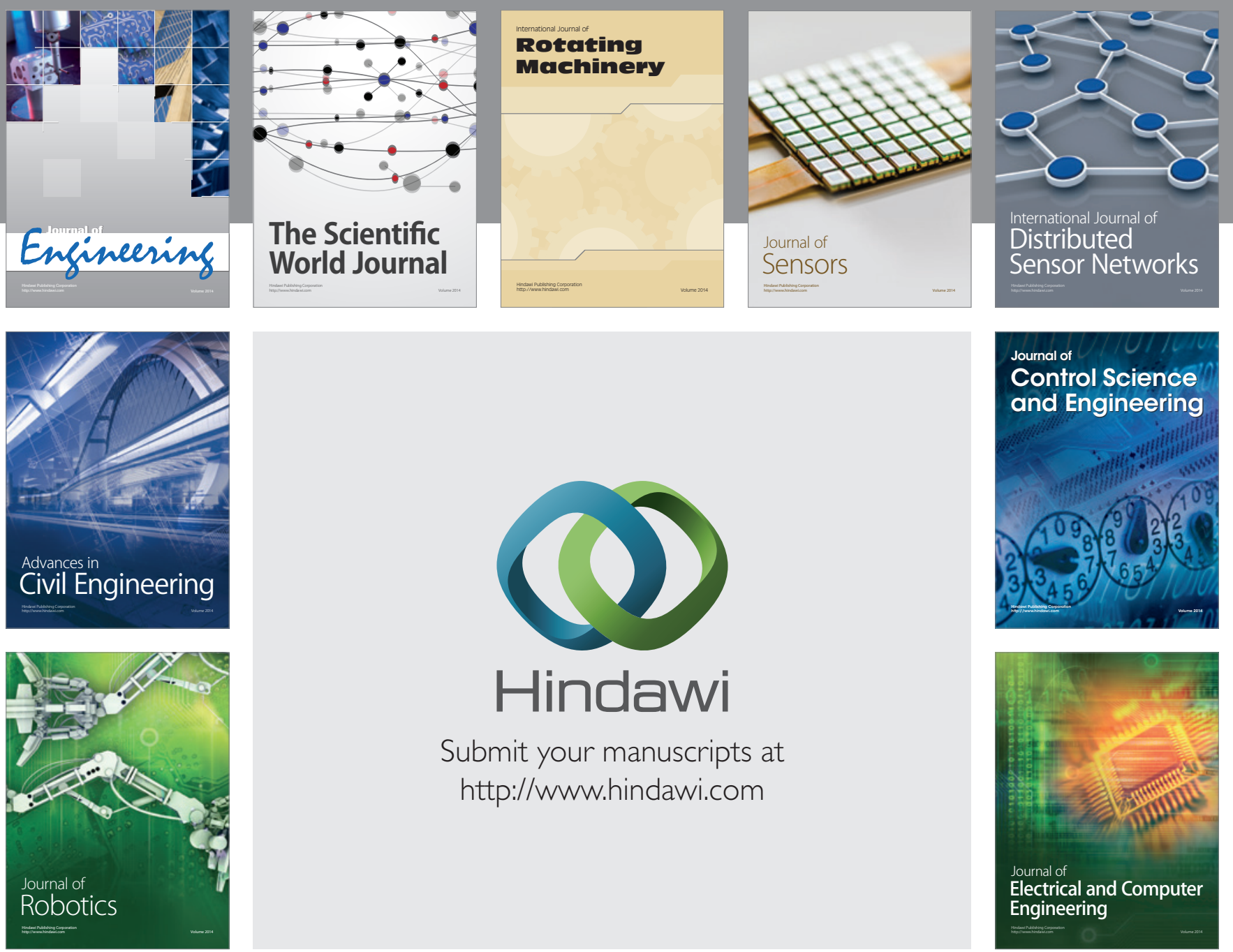

Submit your manuscripts at

http://www.hindawi.com
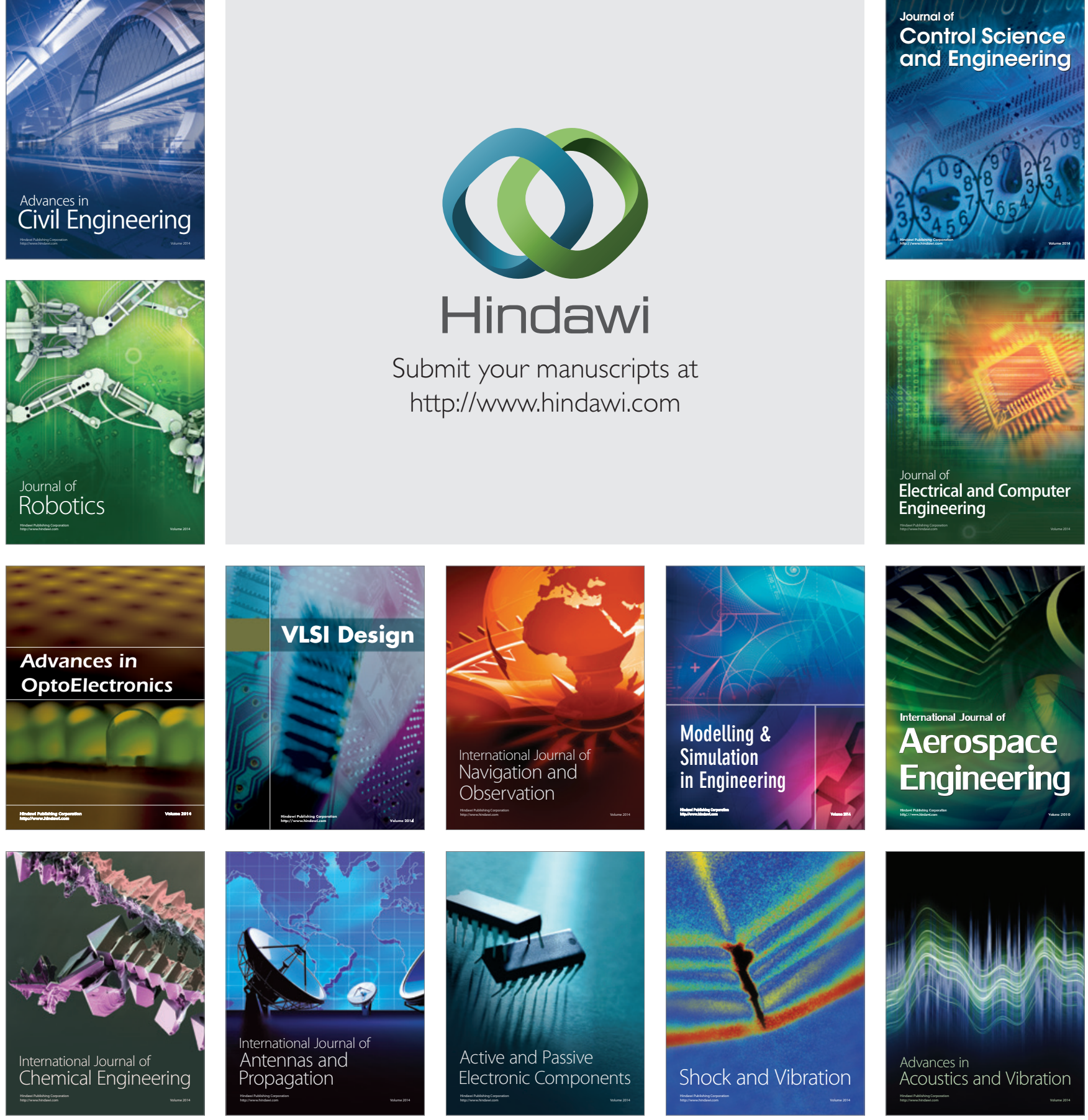